\title{
Methods for measuring the citations and productivity of scientists across time and discipline
}

\author{
Alexander M. Petersen, Fengzhong Wang, and H. Eugene Stanley \\ Center for Polymer Studies and Department of Physics, Boston University, Boston, Massachusetts 02215, USA \\ (Received 6 November 2009; revised manuscript received 23 February 2010; published 24 March 2010)
}

\begin{abstract}
Publication statistics are ubiquitous in the ratings of scientific achievement, with citation counts and paper tallies factoring into an individual's consideration for postdoctoral positions, junior faculty, and tenure. Citation statistics are designed to quantify individual career achievement, both at the level of a single publication, and over an individual's entire career. While some academic careers are defined by a few significant papers (possibly out of many), other academic careers are defined by the cumulative contribution made by the author's publications to the body of science. Several metrics have been formulated to quantify an individual's publication career, yet none of these metrics account for the collaboration group size, and the time dependence of citation counts. In this paper we normalize publication metrics in order to achieve a universal framework for analyzing and comparing scientific achievement across both time and discipline. We study the publication careers of individual authors over the 50-year period 1958-2008 within six high-impact journals: CELL, the New England Journal of Medicine (NEJM), Nature, the Proceedings of the National Academy of Science (PNAS), Physical Review Letters (PRL), and Science. Using the normalized metrics (i) "citation shares" to quantify scientific success, and (ii) "paper shares" to quantify scientific productivity, we compare the career achievement of individual authors within each journal, where each journal represents a local arena for competition. We uncover quantifiable statistical regularity in the probability density function of scientific achievement in all journals analyzed, which suggests that a fundamental driving force underlying scientific achievement is the competitive nature of scientific advancement.
\end{abstract}

DOI: 10.1103/PhysRevE.81.036114 PACS number(s): 89.90. $+\mathrm{n}, 01.85 .+\mathrm{f}, 02.50 .-\mathrm{r}, 01.75 .+\mathrm{m}$

\section{INTRODUCTION}

The study of human success is difficult because information has traditionally been recorded for only the excellent, while individuals with lower than average careers are generally neglected in the record books. Hence, drawing conclusions based only on the relatively few stellar careers will suffer to some extent from selection bias. In contrast, conclusions drawn from the entire population might better illustrate the mechanisms of success. While it is not feasible to obtain the career publication data for every scientist in every journal, it is possible to study a subset of scientists that succeed in publishing in a specific journal.

Several empirical studies have analyzed the citation statistics of individual papers [1-5], of individual journals/fields [5-11], and of subsets of individuals [5,12-14]. In this paper, we study the cumulative citation statistics of individual scientists over their publication careers within a given journal. Studying the distribution of career accomplishment in a particular journal serves as a proxy for the more difficult task of studying the citation statistics of all individuals in all journals, where such all-encompassing data are not as readily available. Here we adopt the working hypothesis that studying publications in high-impact journals offers crude approximation to an author's scientific contribution.

We develop a simple method for normalizing citations so that they can be compared across time and discipline. In order to compare across time, we normalize the number of citations for each paper in a given year by the average number of citations to papers from the same publication year. This rescaling can also aid in removing discipline-specific citation patterns that vary across discipline, especially when considering discipline-specific journals. We further remove discipline-specific collaboration patterns by dividing the achievement equally among the collaboration group members.

This work aims to demonstrate the importance of properly normalizing any conceivable metric that quantifies career achievement (e.g., the citation count, h-index). Extending the work of Radicchi et al. [8], which normalizes the citation values of single articles across discipline by rescaling to local citation averages, our goal is to provide a framework for normalizing the scientific achievement of individual careers. The methodology developed in this paper should conceivably make possible the comparison of careers between various fields. Furthermore, we are able to study the mechanisms of human success in scientific arenas, where effective competition arises from limited financial, temporal, and creative resources.

In addition to studying the distribution of success and productivity, in this paper we also investigate the waiting time between successive achievements, which is intrinsically related to the underlying mechanism of progress. Recent work in $[15,16]$ demonstrates that the Matthew effect (the "rich-get-richer" effect) can be quantified by analyzing the career longevity of employees within competitive professions, such as professional sports and academia. Here, we demonstrate the Matthew effect on the scale of individual authors by analyzing the time intervals between successive publications in high-impact journals. The Matthew effect [17] derives from a passage in the Gospel of Matthew and is a popular conceptual theory in sociology. This theory is analogous to several other positive feedback or cumulative advantage theories $[18,19]$ which have been used to explain the ubiquity of right-skewed distributions that arise in socioeconomic studies. Of particular note, the generic preferential attachment mechanism is relevant to the dynamics of cita- 
tions $[20,21]$ as well as the dynamics of human sexual networks [22].

First, we briefly summarize several results which are relevant to the analysis of success and productivity performed in this paper. A seminal study performed over 50 years ago by Shockley [23] studied the rate of productivity measured by the total number of publications and the total number of patents filed at several large research institutions. In this paper, Shockley suggests that normalizing metrics for output (e.g., patents, papers, citations) by the number of individuals could alleviate the discrepancy between disciplines. In this paper, we normalize metrics for output by the number of contributors such that they are weighted "shares," a procedure recently employed in the calculation of h-index values [14].

Laherrère et al. [12] analyze the top 1120 most-cited physicists over the 16-year period 1981-1997, with the result that the distribution of cumulative citation counts among these scientists, without any normalization procedure, is described by a stretched exponential probability density function (pdf). Redner [1] analyzes approximately 800000 individual papers and found that the pdf $P(x)$ of citations per paper $x$ follows an approximate inverse-cubic power law. This result is found by analyzing the Zipf plot of the number of citations to a particular paper. Interestingly, we find that this result is maintained even after the normalization procedure developed in this paper. Redner [2] also analyzes 110 years of citation statistics in Physical Review journals, where he calculates the citation distribution of 353268 papers, and finds a log-normal pdf $P(x)$ (without normalizing for publication time). In addition to the size and coverage of the Physical Review database, another impressive feature of this study is the analysis of citation dynamics, relating the citation growth rate to the number of contemporaneous citations. Of particular note, Redner finds an approximately linear citation (attachment) rate for citations originating from within Physical Review publications. Also, a recent study [10] analyzes the citation dynamics in 2267 journals and finds that the time-dependent average number of citations per paper within each journal approaches a steady state value which can be used as a normalizing factor for comparing journals across discipline. In this paper, we use the time-dependent average number of citations per paper for a particular journal and publication year as the normalizing factor in order to compare articles across time and discipline.

Recently, Hirsch [13] proposed the h-index to be an unbiased metric to quantify scientific impact. The h-index is calculated using the raw number of citations for each of an author's papers. Although simple in its definition, the h-index has encountered scrutiny, with the opposition claiming that the definition of the h-index is biased in that it neglects differences in publication patterns between scientific (sub)disciplines. It is further biased in that it neglects variations in the size of collaborations, and hence, the credit associated with a given publication. In Ref. [14], Batista et al. normalize the h-index to the number of authors contributing to each paper in order to account for differences in publication styles across discipline. Two additional studies suggest that normalizing by the size of the field can alleviate the differences in research and publication style across disciplines $[7,8]$, how- ever the relationship between citation trends and field size is not trivial and depend on several factors [11]. Very recently, an enormous study of 25 million papers by Wallace et al. [4] implements Tsallis statistics to investigate the distribution of citations for papers spanning the 106-year period 1900 2006. The extensive analysis in [4] also discusses the changes in citation trends over time and the "uncited" phenomena.

Our main result is to provide the first study that quantifies the career publication statistics of individual authors while normalizing the publication statistics with respect to two factors:

(i) the number of authors credited for a particular publication;

(ii) the time-dependent increase of citation counts.

Specifically, we account for (i) by normalizing citation counts and paper tallies to the number of contributing authors, and (ii) by normalizing citations by the local average number of citations per paper; the local average is computed from the set of papers published in the same journal in the same year. While these two factors have been discussed, to our knowledge, no study has incorporated them both simultaneously.

In order to compare careers that are of similar duration, we use methods described in [24] to isolate "completed" careers from our journal databases, which all span the 50-year period 1958-2008 except for CELL which was created only in 1974. For the subset of careers that meet a completion criterion, we normalize each individual citation according to factors (i) and (ii). We then tally the normalized citation shares for each scientist, which serves as one possible metric for career accomplishment. We also perform the analogous procedure for paper shares which serves as a metric for career productivity.

The organization of this paper is as follows: in Sec. II we review the data analyzed, the procedure with which we aggregate the data into publication careers, and the possible systematic errors inherent in our method. In Sec. III we analyze the distribution of both citation and productivity statistics for three high-impact multidisciplinary journals: Nature, the Proceedings of the National Academy of Science (PNAS), and Science, and also for three less multidisciplinary journals: CELL, the New England Journal of Medicine (NEJM), and Physical Review Letters (PRL). We note that only three of these journals analyzed are discipline specific, and so we rely significantly on the results of $[7,8]$ in justifying the comparison of normalized career metrics across discipline beyond our results for the high-impact journals CELL, the New England Journal of Medicine (NEJM), and Physical Review Letters (PRL).

\section{DATA AND METHODS}

We downloaded journal data in May 2009 from ISI Web of Knowledge [25]. We restrict our analysis to publications termed "Articles," which excludes reviews, letters to editors, corrigendum, etc. For each journal, we combine all publications into one database. In total, these data represent approximately 350000 articles and 600000 scientists (see Table I). 
TABLE I. Summary of data set size for each journal. Total number $N$ of unique (but possibly degenerate) name identifications.

\begin{tabular}{lccc}
\hline \hline Journal & Years & Articles & Authors, $N$ \\
\hline CELL & $1974-2008$ & 53290 & 31918 \\
NEJM & $1958-2008$ & 17088 & 66834 \\
Nature & $1958-2008$ & 65709 & 130596 \\
PNAS & $1958-2008$ & 84520 & 182761 \\
PRL & $1958-2008$ & 85316 & 112660 \\
Science & $1958-2008$ & 48169 & 109519 \\
\hline \hline
\end{tabular}

Our data collection procedure begins with downloading all "articles" for each journal for year $y$ from ISI Web of Knowledge. From the set of $N(y)$ articles for each particular journal and year, we calculate $\langle c(y)\rangle$, the average number of citations per article at the date of data extraction (May 2009). Each article summary includes a field for a contributing author's name identification, which consists of a last name and first and middle initial [26]. From these fields, we aggregate the career works of individual authors within a particular journal. In this paper we develop normalized metrics for career success and productivity, while in [16] we compare theory and empirical data for career longevity.

For each author, we combine all his/her articles in a given journal. Specifically, a publication career in this paper refers to the lifetime achievements of a single author within a single journal, and not the lifetime achievements combined among the six journals analyzed. We define $n$ as the total number of papers for a given author in a given journal over the 50-year period. In analogy with the traditional citation tally, one can calculate the career success/impact within a given journal by adding together the citations $c_{i}$ received by the $n$ papers,

$$
C=\sum_{i=1}^{n} c_{i}
$$

Furthermore, one can calculate the career productivity of a given author within a specific journal as the total number $P$ of papers published within the journal. A main point raised in this paper is to discount the value of citation metrics which do not take into account the time evolution of citation accumulation.

Naturally, some older papers will have more citations than younger papers only because the older papers have been in circulation for a longer time. In Fig. 1 we plot $\langle c(y)\rangle$, the average number of citations for articles from a given year, and confirm that the time dependence of citation accumulation is an important factor. Interestingly, it is found in [10] that the pdf of citations from papers within a given year and journal is approximately log normal, where the average value of the distribution has a time-dependent drift. With increasing time, the pdf approaches a steady state distribution which is also approximately log normal. Hence, the nonmonotonicity in $\langle c(y)\rangle$ suggests that an important factor in the dynamics of citation counts is the growth with time of the scientific body and the scientific output. The mechanism underlying

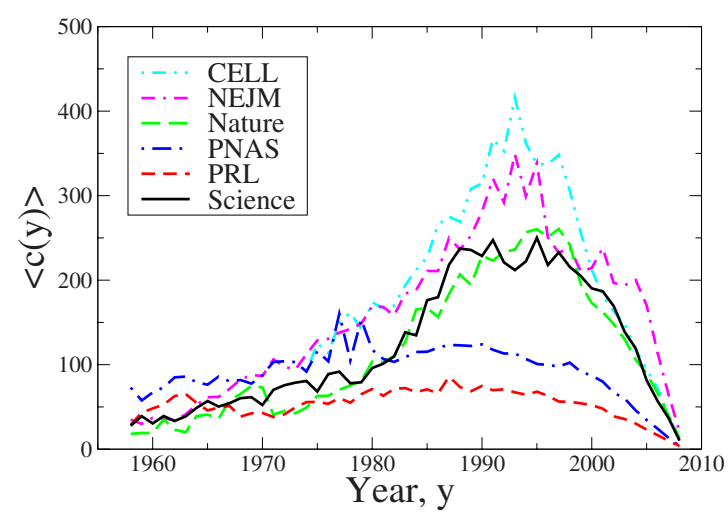

FIG. 1. (Color online) The average number of citations $\langle c(y)\rangle$ per article for each journal in year $y$ demonstrates the time dependence of citations. This quantity serves as a normalizing factor, so that we can detrend citation values across different years. The popular Impact Factor (IF) [10,11] of a journal for a particular year is the average number of citations obtained in a given year for articles published over the previous two years. In this paper we restrict our analysis to journals with large IF, ensuring that there is considerable competition for limited publication space in such journals.

the evolution of citation trends and impact factors is complex, where it is found that citation growth rates decompose into several components in addition to the growth of science [11]. Another criticism of Eq. (1) is that it does not take into account the variability in number of coauthors, which varies both within and across discipline (see Fig. 3).

To remedy these problems, we propose a simple success metric termed citation shares, which normalizes the citations $c_{i}(y)$ of paper $i$ by $\langle c(y)\rangle$, the average number of citations for papers in a given journal in year $y$, and divides the quantity $c_{i}(y) /\langle c(y)\rangle$ into equally distributed shares among the $a_{i}$ coauthors. Dividing the shares equally will obviously discount the value of the efforts made by greater contributors while raising the value of the efforts made by lesser contributors. Without more accurate reporting schemes on the extent of each authors' contributions (as is now implemented in e.g., Nature and PNAS), dividing the shares equally is the most reasonable method given the available data. Hence, we calculate the normalized career citation shares as

$$
C_{s}=\sum_{i=1}^{n} \frac{1}{a_{i}} \frac{c_{i}(y)}{\langle c(y)\rangle} .
$$

An analogous estimator for career productivity is $P_{s}$, the total number of paper shares within a given journal,

$$
P_{s}=\sum_{i=1}^{n} \frac{1}{a_{i}}
$$

which partitions the credit for each publication into equal shares among the $a_{i}$ coauthors.

There is another sampling bias that we address. Currently, we assume that all careers are comparable in their duration, or more precisely, maturity. However, without further consideration, this assumption would ensure that we are comparing the careers of graduate students with seasoned professors. 
TABLE II. Summary of citation shares for "completed" careers. The reduced size of the data set has size $N^{*}$. The average number of citation shares $\left\langle C_{s}\right\rangle$ for careers within each journal are computed from the subset of "completed" careers (the value in parenthesis corresponds to value for all careers). The value of the power-law exponent $\beta$ corresponds to the Zipf plot of citation shares plotted in Fig. 5, where we calculate the value of $\beta$ using data in the range $10<$ rank $<N_{M L E}$ implementing a linear regression on a log-log scale, where $N_{M L E}$ is the number of data values used to calculate $\alpha$. The value of the power-law exponent $\alpha$ corresponds to the pdf of citation shares plotted in Fig. 4(b), where we calculate the value of $\alpha$ using Hill's maximum likelihood estimator for data values greater than a cutoff $C_{s}^{c} \equiv 1$.

\begin{tabular}{lrccc}
\hline \hline Journal & \multicolumn{1}{c}{$N^{*}$} & $\left\langle C_{s}\right\rangle$ & $\beta$ & $\alpha$ \\
\hline CELL & 23060 & $0.34(0.35)$ & $0.52 \pm 0.01$ & $2.60 \pm 0.04$ \\
NEJM & 49341 & $0.25(0.26)$ & $0.45 \pm 0.01$ & $2.65 \pm 0.04$ \\
Nature & 94221 & $0.46(0.50)$ & $0.56 \pm 0.01$ & $2.42 \pm 0.02$ \\
PNAS & 118757 & $0.42(0.46)$ & $0.57 \pm 0.01$ & $2.50 \pm 0.02$ \\
PRL & 72102 & $0.61(0.75)$ & $0.55 \pm 0.01$ & $2.25 \pm 0.02$ \\
Science & 82181 & $0.43(0.44)$ & $0.56 \pm 0.01$ & $2.43 \pm 0.02$ \\
\hline \hline
\end{tabular}

Hence, we implement a standard method to isolate "completed" careers from our data set which begins at year $Y_{0}$ and ends at year $Y_{f}$, a common method described in [24]. For each author $z$ we calculate $\left\langle\Delta \tau_{z}\right\rangle$, his/her average time between successive publications in a particular journal. A career which begins with the first recorded publication in year $y_{z, 0}$ and ends with the final recorded publication in year $y_{z, f}$ is considered "complete" if the following two criteria are met:

(i) $y_{z, f} \leq Y_{f}-\left\langle\Delta \tau_{z}\right\rangle$ and

(ii) $y_{z, 0} \geq Y_{0}+\left\langle\Delta \tau_{z}\right\rangle$.

In other words, this method estimates that the career begins in year $y_{z, 0}-\left\langle\Delta \tau_{z}\right\rangle$ and ends in year $y_{z, f}+\left\langle\Delta \tau_{z}\right\rangle$. If either the beginning or ending year do not lie within the range of the database, then we discount the career as incomplete to first approximation. Statistically, this means that there is a significant probability that this author published before $Y_{0}$ or will publish after $Y_{f}$. Using this criterion reduces the size of the data set by approximately $25 \%$ (compare the raw data set sizes $N$ in Table I to the data set sizes $N^{*}$ in Table II). The results reported in this paper are, unless otherwise stated, based on the analysis of only the subset of "completed" careers. Another justification for this criterion is that we recently implement this method in previous work on career longevity in [16], which is more sensitive to using or not using the criterion.

We note some potential sources of systematic error in the use of this database:

(1) Degenerate names leads to misleading increases in career totals.

(2) Authors using middle initials in some but not all instances of publication decreases career totals.

(3) A midcareer change of (last) name decreases career totals.
(4) Sampling bias due to finite time period. Recent young careers are biased toward short careers. Long careers located toward the beginning $Y_{i}$ or end $Y_{f}$ of the database are biased toward short careers and hence decrease career totals.

Radicchi et al. [5] observe that the method of concatenated author ID leads to a pdf $P(d)$ of degeneracy $d$ that scales as $P(d) \sim d^{-3}$, which contributes to the systematic error mentioned in item 1 . Although the size of our data set guarantees almost surely that such errors exist (given the prevalence of last names Wang, Lee, Johnson, etc.), these errors should be negligible in the estimation of pdf parameters quantifying a significant portion of the data set.

\section{RESULTS}

\section{A. Individual papers}

The growth dynamics of citations vary, ranging from stunted growth to steady growth and, in some cases where research is published ahead of its time, to late blooming growth [2]. One objective of this paper is to account for the time dependence of citation counts in a consistent way so that citations can be compared across time. We detrend citation counts to a time-independent framework by dividing the number of citations a paper has received by the average number of citations for all papers published in the given journal in the same year. In Fig. 1 we plot $\langle c(y)\rangle$, the average number of citations per paper, where the average is performed over the full set of papers in each given journal for each year $y$. We note that $\langle c(y)\rangle$ approaches zero as the year becomes contemporaneous with the data download date, and that the peak value of $\langle c(y)\rangle$ occurs for papers published approximately 15-20 years before our data download date in 2009. The presence of this maximum value reflects the growth of the scientific body, the growth of scientific productivity, and the time delay over which ideas become relevant and established. See Ref. [10] for the average citation profiles $\langle c(y)\rangle$ of 2266 journals indexed by ISI. Normalizing to this standard baseline allows one to compare the success of papers across scientific disciplines, first demonstrated in Ref. [8].

In order to visualize the effects of normalizing citations to a local average in the case of single papers, we compare the un-normalized cumulative distribution functions (cdfs) of Fig. 2(a) with the normalized cdf of Fig. 2(b). The procedure of normalizing by the local average reduces the variations across journal (discipline [5]), revealing a universal scaling law $P(x>q) \sim q^{1-\gamma}$ with $\gamma-1 \approx 2$. The scaling exponent $\gamma$ $\approx 3$ describing the success of individual papers was first reported in [1], where normalizing techniques were not employed. Surprisingly, we observe the same value of $\gamma$ here for the normalized citation statistics of individual papers from several major journals over a 50-year period.

In addition to time-dependent factors, we also consider factors resulting from various research styles across the broad range of scientific disciplines. In science, the resources required to make significant scientific advances range from a pencil and paper to million-dollar laboratory equipment. Similarly, the number of contributors to scientific advances ranges from a single scientist to projects involving several hundred scientists. Figure 3 illustrates the pdf of collabora- 

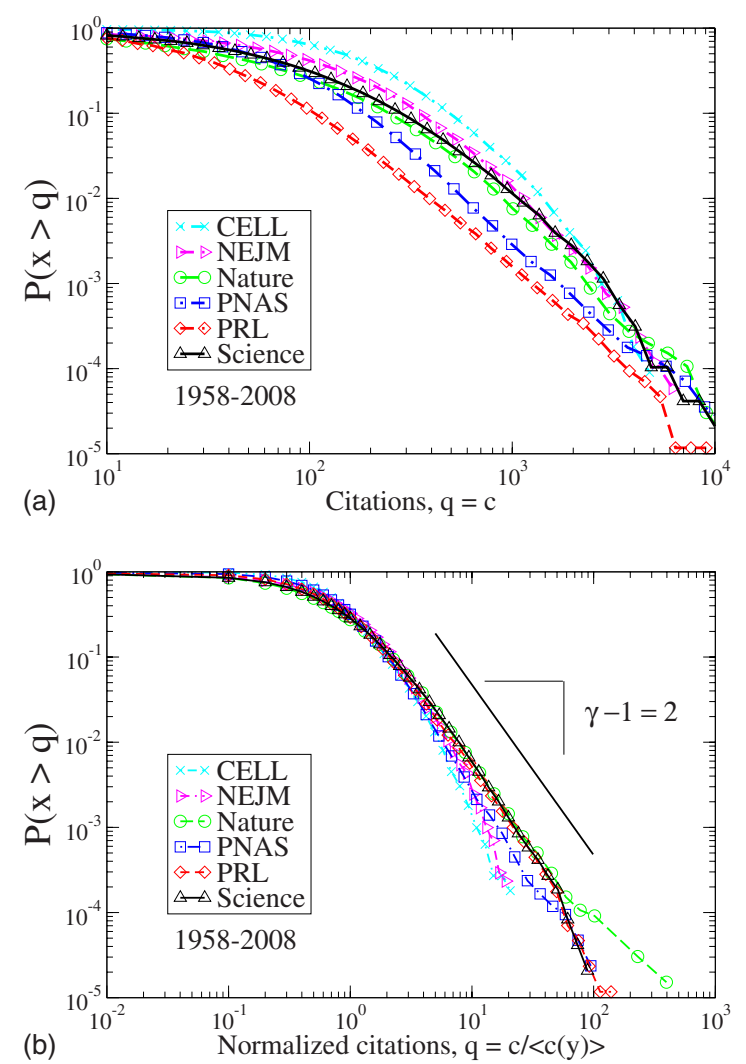

FIG. 2. (Color online) Data collapse in the distribution of article citations for several journals is achieved by accounting for the time dependence of citations. (a) The CDF of raw citations $c$ depends on the field. (b) Normalizing the number of citations $c$ by $\langle c(y)\rangle$, the average number of citations for a particular year in a particular journal, the CDF for different journals are remarkably similar, with the power law with $\gamma-1 \approx 2$. We calculate $\gamma$ for each journal using Hill's MLE and obtain values for the scaling exponent corresponding to each journal: $\gamma=3.64 \pm 0.12$ (CELL), 3.31 \pm 0.07 (NEJM), $2.87 \pm 0.03$ (Nature), $3.30 \pm 0.04 \quad(P N A S), 2.96 \pm 0.03$ (PRL), $2.86 \pm 0.03$ (Science). We provide a power law (solid line) with exponent $\gamma-1=2$ for reference.

tion size associated with a single publication. The pdf is significantly right skewed, especially for publications in NEJM and PRL where occasionally, the number of authors contributing to a single publication exceeds 100 individuals. For instance, in the cases of research at major medical institutes and particle accelerators, it is common for the credit for the scientific advance reported in a publication to be shared by extremely large numbers of contributors. In Eqs. (2) and (3) we choose a simple weighting recipe for associating credit among $a_{i}$ authors of a single paper $i$. We assign equal credit for all authors. Although this recipe may grant some authors more credit than due, it also credits other authors with less credit than due. We believe this weighting scheme is useful in proportionally sharing the credit for a scientific advancement among the $a_{i}$ authors. To address this issue, the journals Nature and PNAS require the corresponding author to assign credit to each co-author across a broad range of categories such as theoretical analysis, experimental

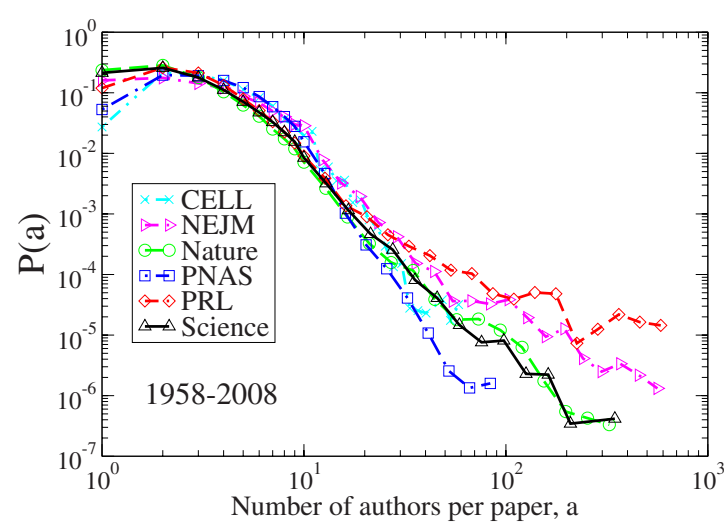

FIG. 3. (Color online) The citations and credit for a publication are typically shared fully among all $a$ co-authors, unless the journal specifically allows for designation of specific credit. The pdf of $a$ for each journal demonstrates that the credit for a single publication can be distributed across a very broad number of contributors. In this paper, we propose normalizing credit into fractional "shares," to account for the variations in collaboration size. The average number of authors contributing to an article in each journal are $\langle a\rangle$ =4.8 (CELL), 5.9 (NEJM), 3.5 (Nature), 4.6 (PNAS), 9.1 (PRL), 3.7 (Science).

methods, and writing of the manuscript. If adopted across all journals, this formalism could potentially improve the quantitative allocation of scientific credit, thus improving the quantitative measures for individual scientific impact.

\section{B. Citation shares}

In Figs. 4(a) and 4(b) we present the pdfs of career citations $C$ and of career citation shares $C_{s}$, corresponding to Eq. (1) and Eq. (2), respectively. While the six pdfs of $C$ in Fig. 4(a) are all similarly right skewed, the collapse onto a universal function is weak for small values of $C$.

The discrepancy between the pdf curves for small and large citation counts in Fig. 4(a) is likely associated with factors associated with the size of the scientific field, the size of the collaboration group and the impact of the research. Since we study only six high-impact journals, these factors should be negligible in the overall difference across discipline and journal, since we assume both discipline and journal are large. Hence, the collapse of the six pdfs for normalized citation shares in Fig. 4(b) demonstrates that normalization is necessary. For each pdf $P\left(C_{s}\right)$ we observe a scaling regime

$$
P\left(C_{s}\right) \sim C_{s}^{-\alpha},
$$

and we estimate the scaling exponent $\alpha$ in the tail of the pdf using the maximum likelihood estimator (MLE), also known as the Hill estimator [27,28].

We list $\alpha$ values calculated for $C_{s}>C_{s}^{c} \equiv 1$ in Table II. The Hill estimator is a robust method for approximating power-law exponents which incorporates each data observation $C_{s}^{i}$ greater than a cutoff value $C_{s}^{c}$ into the calculation of $\alpha$, 

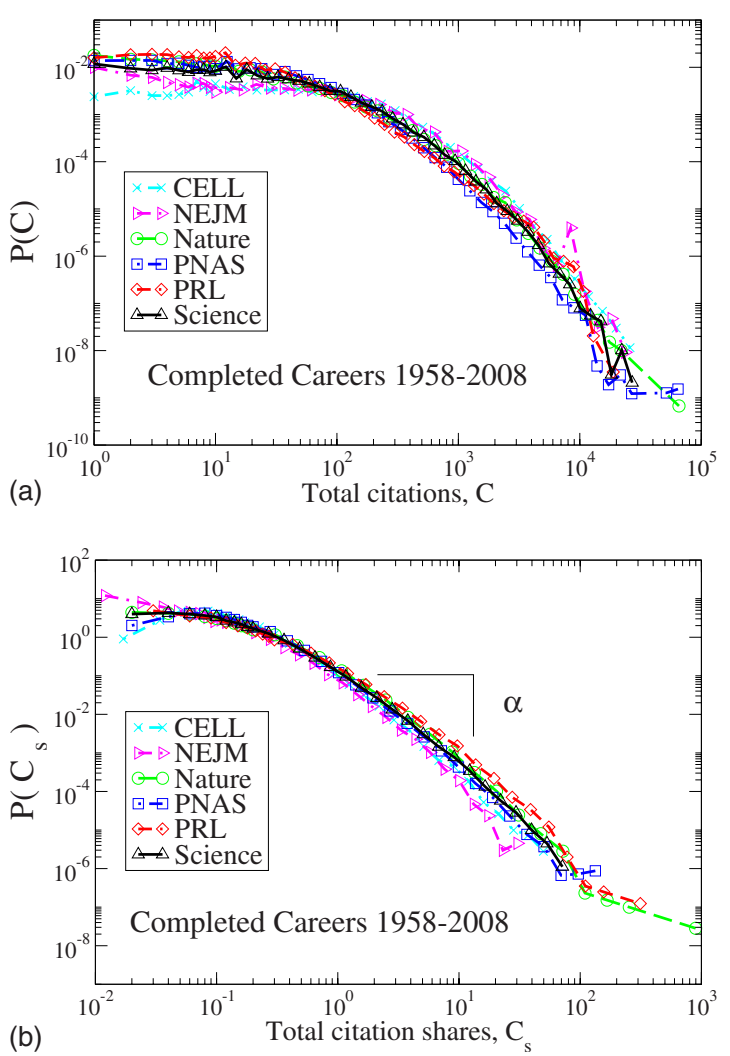

FIG. 4. (Color online) We estimate the career success of a scientist within a given journal using the citation shares metric $C_{s}$ defined in Eq. (2), which accounts for both the number of authors and the age of the paper. (a) PDF of total raw citations $C$ according to Eq. (1) for "completed" careers. (b) PDF of total citation shares $C_{s}$ according to Eq. (2) for "completed" careers. A given career is considered "complete" if there is a large likelihood that the data set contains all of the particular author's publications. The normalization procedure results in significant data collapse in panel (b), with the value of the scaling exponent $\alpha \approx 2.5$ for all journals analyzed.

$$
\alpha=1+\frac{N}{\sum_{i} \ln \left(C_{s}^{i} / C_{s}^{c}\right)},
$$

with standard error,

$$
\delta \alpha \approx(\alpha-1) / \sqrt{N}
$$

For each journal, the number of data points $N$ greater than $C_{s}^{c}$ used in the calculation of $\alpha$ is approximately $10 \%$ of the total data set size $N^{*}$. Remarkably, the scaling exponent for citation statistics of completed careers is approximately 2.5 for all journals analyzed. Hence, we find convincing evidence for a universal scaling function representing the distribution of citation shares for scientific careers in competitive highimpact journals. Interestingly, the values of $\alpha$ for each journal are less than the values of $\gamma \approx 3$ which describes the scaling of normalized single article citation counts in Fig. 2. This result implies that the success of individuals over their entire careers is not related in a simple way to the success of a random number of independent articles. Instead, there is a

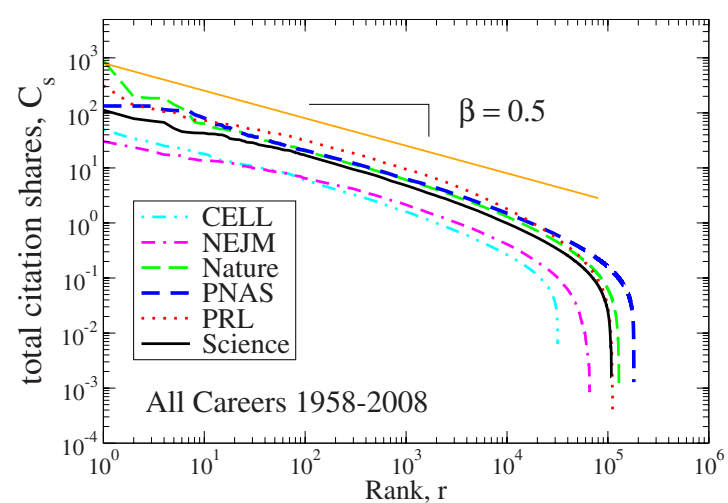

FIG. 5. (Color online) The Zipf plot emphasizes the stellar careers corresponding to large $C_{s}$, the total number of citation shares within a particular journal defined in Eq. (2), and shows a significant scaling regime corresponding to the top-ranking "champions" of each journal. For comparison, we list the top 20 careers within the journals CELL, NEJM, and PRL in Table III. The total number of career citation shares for a particular author in a given journal serves as a proxy for the career success of the scientist. The statistical regularity in the rank ordering of scientific achievement extends over four orders of magnitude. The similarity in scaling exponent among the journals analyzed possibly suggests that there are fundamental forces governing success in competitive arenas such as high-impact journals. For visual clarity, we plot the power law with scaling exponent $\beta \equiv 0.5$.

larger number of stellar careers than would be expected from the number of stellar papers.

Another illustrative method for comparing the distribution of success across the entire range of individuals is the popular Zipf plot, which is mathematically related to the pdf $[1,9]$. In Fig. 5 we plot $C_{s}$ versus rank for the same set of completed careers analyzed in Fig. 4(b). The Zipf plot emphasizes the scaling in the tail of the pdf, which is represented by high rank values. We calculate the scaling exponent of the Zipf plot for rank values in the range $10<r<r_{c}$ for each journal, where $r_{c}$ corresponds to the number of data points incorporated into the calculation of $\alpha$ using Hill's MLE. These values are in approximate agreement with the expected relationship $1+1 / \beta \approx \alpha$.

The small range of $\beta$ values across journals (see Table II) demonstrates that our normalization procedure places scientific accomplishments on a comparable footing across both time and discipline. In Table III we list the top 20 publication careers according to citation shares. This table consists mostly of careers that have many papers of significant impact; however, it also contains a few careers that are distinguished by a small number of seminal papers. Hence, while longevity at the upper tier of science is good at assuring reputation and success, there are also a few instances of success achieved via a singular yet monumental accomplishment.

\section{Paper shares}

We now focus on scientific productivity, quantified by the number of papers published by a given author. In Fig. 6 we plot the pdfs for paper shares defined in Eq. (3). In order to 
TABLE III. The top 20 authors (not necessarily "completed") in the journals CELL, NEJM, and PRL, ranked according to citation shares $C_{s}$ accumulated from their $n$ papers published in each journal. Our normalization procedure offers one way to quantitatively order the top authors.

\begin{tabular}{|c|c|c|c|c|c|c|c|c|}
\hline \multicolumn{3}{|l|}{ CELL } & \multicolumn{3}{|l|}{ NEJM } & \multicolumn{3}{|l|}{ PRL } \\
\hline Name & $C_{s}$ & $n$ & Name & $C_{s}$ & $n$ & Name & $C_{s}$ & $n$ \\
\hline GREEN, H & 49.7 & 35 & BRAUNWALD, E & 30.3 & 59 & WEINBERG, S & 313.3 & 49 \\
\hline BALTIMORE, D & 33.8 & 64 & KOCHWESER, J & 23.6 & 28 & ANDERSON, PW & 137.4 & 64 \\
\hline MANIATIS, T & 29.5 & 55 & MCCORD, JM & 20.2 & 1 & WILCZEK, F & 120.0 & 62 \\
\hline SHARP, PA & 25.1 & 41 & FINLAND, M & 17.4 & 36 & TERSOFF, J & 105.1 & 76 \\
\hline TJIAN, R & 23.8 & 45 & HENNEKENS, CH & 16.9 & 36 & HALDANE, FDM & 102.3 & 38 \\
\hline LEDER, $\mathrm{P}$ & 22.4 & 39 & REICHLIN, S & 16.7 & 10 & YABLONOVITCH, E & 87.5 & 21 \\
\hline AXEL, R & 20.9 & 52 & VECCHIO, TJ & 14.8 & 1 & PERDEW, JP & 78.3 & 20 \\
\hline WEINTRAUB, H & 20.5 & 46 & STAMPFER, MJ & 14.3 & 45 & LEE, PA & 74.6 & 76 \\
\hline KARIN, M & 18.5 & 40 & TERASAKI, PI & 13.7 & 29 & PENDRY, JB & 74.1 & 29 \\
\hline RUBIN, GM & 18.0 & 52 & OSSERMAN, EF & 13.7 & 6 & PARRINELLO, M & 72.8 & 68 \\
\hline KOZAK, M & 17.1 & 6 & KUNIN, CM & 13.5 & 16 & FISHER, ME & 71.6 & 67 \\
\hline ROEDER, RG” & 15.5 & 44 & YUSUF, S & 13.4 & 18 & CIRAC, JI & 66.7 & 97 \\
\hline RHEINWALD, JG & 14.7 & 7 & ROSEN, FS & 13.2 & 42 & HALPERIN, BI & 66.7 & 50 \\
\hline EVANS, RM & 14.1 & 32 & CHALMERS, TC & 13.1 & 30 & RANDALL, L & 63.4 & 14 \\
\hline OFARRELL, PH & 13.9 & 14 & AUSTEN, KF & 12.9 & 30 & BURKE, K & 63.2 & 18 \\
\hline GLUZMAN, Y & 13.3 & 2 & WELLER, TH & 12.7 & 7 & JOHN, S & 62.8 & 20 \\
\hline HUNTER, T & 13.2 & 27 & GARDNER, FH & 12.6 & 19 & GEORGI, H & 61.9 & 26 \\
\hline GOLDSTEIN, JL & 13.0 & 36 & DIAMOND, LK & 12.6 & 18 & CAR, R & 59.8 & 51 \\
\hline PENMAN, S & 12.9 & 30 & FEINSTEIN, AR & 12.2 & 16 & GLASHOW, SL & 59.6 & 37 \\
\hline BROWN, MS & 12.8 & 35 & MERRILL, JP & 11.9 & 25 & CEPERLEY, DM & 58.9 & 39 \\
\hline
\end{tabular}

collapse the pdfs for the six journals analyzed, we hypothesize that a universal function for productivity can be written as $P\left(P_{s}\right)=f\left(P_{s} /\left\langle P_{s}\right\rangle\right)$. In an effort to compare the pdfs across discipline, we approximate the generic pdf of paper shares by a log-normal distribution with a heavy tail after a cutoff value $P_{s}^{c}$. Quantitatively, we represent the general form of the pdf as

$$
P\left(P_{s}\right) \propto\left\{\begin{array}{ll}
\frac{1}{P_{s}} \exp \left[-\left(\ln P_{s}-\mu\right)^{2} / 2 \sigma^{2}\right] & P_{s} \lesssim P_{s}^{c} \\
P_{s}^{-\alpha} & P_{s} \approx P_{s}^{c}
\end{array} .\right.
$$

The least-square parameters for the log-normal fit and the MLE parameter for the scaling regime are listed in Table IV. The log-normal distribution is consistent with the prediction by Shockley [23] that productivity (as estimated here by paper shares) is a result of a series of multiplicative factors, which can lead to log-normal [29], stretched exponential [30], and even power-law [31] distributions, given the appropriate set of systematic conditions.

\section{Matthew effect}

We conclude this section with quantitative evidence of the "Matthew effect" in the advancement of scientific careers. In Fig. 7 we plot the average waiting time between publications $\langle\tau(n)\rangle$ for all authors that meet the complete career criterion by averaging the difference in publication year for the paper

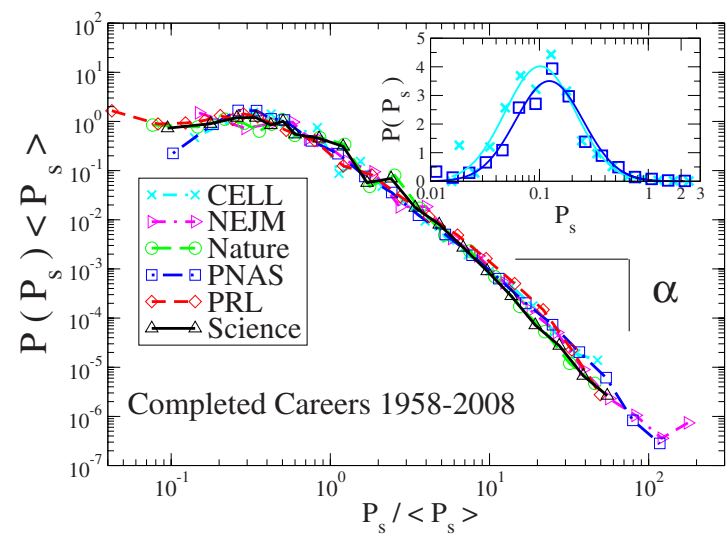

FIG. 6. (Color online) As a proxy for career productivity, we define paper shares $P_{s}$ in Eq. (3), which accounts for variations in the size of the collaboration. In order to collapse the pdfs of total paper shares for completed careers within the six journals analyzed, we hypothesize that the universal scaling function quantifying productivity can be written as $P\left(P_{s}\right)=f\left(P_{s} /\left\langle P_{s}\right\rangle\right)$. We approximate the generic pdf of paper shares by a log-normal distribution with a power-law tail after a cutoff value $P_{s}^{c} \approx 1$. We list the values of the log-normal parameters $\mu$ and $\sigma$, and the scaling parameter $\alpha$ for each journal in Table IV. (Inset) We plot the pdf for CELL and $P N A S$ data on log-linear axes for $P_{s} \leq 3$ in order to demonstrate the log-normal form consistent with the prediction by Shockley [23] that productivity can be modeled as a series of random multiplicative factors. 
TABLE IV. Summary of paper shares for "completed" careers. The value of the log-normal fit parameters $\mu$ and $\sigma$ correspond to the pdf before the cutoff value of $P_{s}^{c} \approx 2$ paper shares. The values of $\alpha$ are calculated using a data values after the cutoff $P_{s}^{c} \equiv 1$ paper shares, which corresponds to approximately $8 \%$ of the total data for each journal.

\begin{tabular}{lccc}
\hline \hline Journal & $\mu$ & $\sigma$ & $\alpha$ \\
\hline CELL & $-1.7 \pm 0.1$ & $0.7 \pm 0.1$ & $2.60 \pm 0.05$ \\
NEJM & $-1.7 \pm 0.1$ & $1.0 \pm 0.1$ & $2.60 \pm 0.02$ \\
Nature & $-1.3 \pm 0.1$ & $1.0 \pm 0.1$ & $2.74 \pm 0.05$ \\
PNAS & $-1.6 \pm 0.1$ & $0.7 \pm 0.1$ & $2.56 \pm 0.02$ \\
PRL & $-1.1 \pm 0.1$ & $1.0 \pm 0.1$ & $2.35 \pm 0.02$ \\
Science & $-1.4 \pm 0.1$ & $0.9 \pm 0.1$ & $2.61 \pm 0.02$ \\
\hline \hline
\end{tabular}

$n$ and the paper $n+1$. The values of $\langle\tau(1)\rangle$ for each journal are 2.2 (CELL, PRL), 3.0 (Nature, PNAS, Science) and 3.5 (NEJM) years. The decrease in waiting time between publications is a signature of the cumulative advantage mechanism qualitatively described in [19] and quantitatively analyzed in $[16,18]$. To avoid presenting statistical fluctuations arising from the small size of data sets, we only present $\langle\tau(n)\rangle$ computed for data sets exceeding 75 observations.

To explain the steady decline of the curve for PRL we mention that PRL has many authors with many articles $(n \gtrsim 100)$. A possible explanation is that a significant number of these authors are involved in large particle accelerator experiments with multiple collaborating groups. These multilateral projects contribute significantly to the heavy tail observed in the pdf of the number of authors per paper (Fig. 3). Hence, the decay in the curve for PRL which approaches zero might be due to the project leaders at large experimental

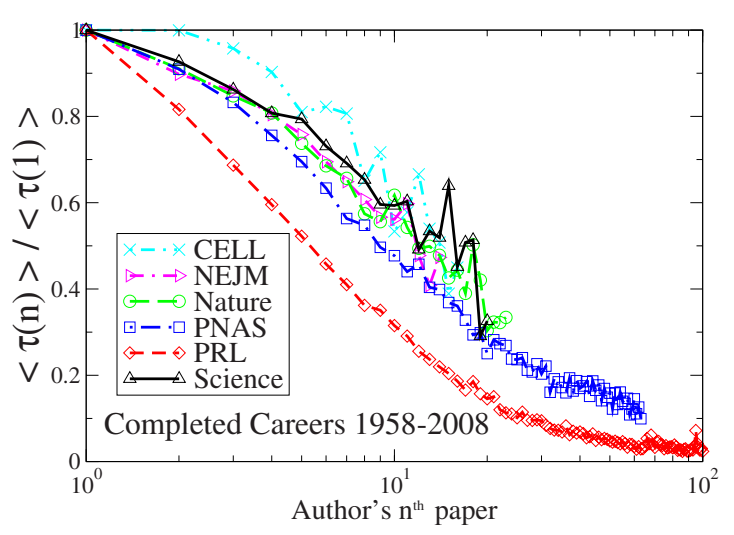

FIG. 7. (Color online) A decreasing waiting time $\tau(n)$ between publications in a given journal suggests that a longer publication career (larger $n$ ) facilitates future publications, as predicted by the Matthew effect. We plot $\langle\tau(n)\rangle /\langle\tau(1)\rangle$, the average waiting time $\langle\tau(n)\rangle$ between paper $n$ and paper $n+1$, rescaled by the average waiting time between the first and second publication, $\langle\tau(1)\rangle$. The values of $\langle\tau(1)\rangle$ are 2.2 (CELL, PRL), 3.0 (Nature, PNAS, Science), and 3.5 (NEJM) years. Physical Review Letters exhibits a more rapid decline in $\tau(n)$, reflecting the rapidity of successive publications (often by large high-energy experiment collaborations), which is possible in this high-impact letters journal. institutions which produce over many years many significant results per year. Furthermore, the organization of the curves in Fig. 7 suggests that it is more difficult at the beginning of a career to repeatedly publish in CELL than PRL. Reaching a crossover point along the career ladder is a generic phenomenon observed in many professions. Accordingly, surmounting this abstract crossover is motivated by significant personal incentives, such as salary increase, job security, and managerial responsibility.

\section{DISCUSSION}

Scientific careers share many qualities with other competitive careers, such as the careers of professional sports players, inventors, entertainers, actors, and musicians $[15,32,33]$. Limited resources such as employment, salary, creativity, equipment, events, data samples, and even individual lifetime contribute to the formation of generic arenas for competition. Hence, of interest here is the distribution of success and productivity in high-impact journals which in principle have high standards of excellence.

In science, there are unwritten guides to success requiring ingenuity, longevity, and publication. We observe a quantifiable statistical regularity describing publication careers of individual scientists across both time and discipline. Interestingly, we find that the scaling exponent for individual papers $(\gamma \approx 3)$ is larger than the scaling exponent for total citation shares $(\alpha \approx 2.5)$ and the scaling exponent for total paper shares $(\alpha \approx 2.6)$, which indicates that there is a higher frequency of stellar careers than stellar papers. This is consistent with the observation that a stellar career can result from an arbitrary combination of stellar papers and consistent success, as demonstrated in Table III. In all, the statistical regularity found in the distributions for both citation shares and paper shares lend naturally to methods based on extreme statistics in order to distinguishing stellar careers. Such methods have been developed for Hall of Fame candidacy in baseball [16,34], where statistical benchmarks are established using the distribution of success.

Statistical physicists have long been interested in complex interacting systems, and are beginning to succeed in describing social dynamics using models that were developed in the context of concrete physical systems [35]. This study is inspired by the long term goal of using quantitative methods from statistical physics to answer traditional questions rooted in social science [36], such as the nature of competition, success, productivity, and the universal features of human activity. Many studies begin as empirical descriptions, such as the studies of common mobility patterns [37], sexuality $[38,39]$, and financial fluctuations [40], and lead to a better understanding of the underlying mechanics. It is possible that the empirical laws reported here will motivate useful descriptive theories of success and productivity in competitive environments.

\section{ACKNOWLEDGMENTS}

We thank S. Miyazima and S. Redner for helpful comments and the NSF for financial support. 
[1] S. Redner, Eur. Phys. J. B 4, 131 (1998).

[2] S. Redner, Phys. Today 58 (6), 49 (2005).

[3] S. Maslov and S. Redner, J. Neurosci. 28, 11103 (2008).

[4] M. L. Wallace et al., Journal of Infometrics 3, 296 (2009).

[5] F. Radicchi, S. Fortunato, B. Markines, and A. Vespignani, Phys. Rev. E 80, 056103 (2009).

[6] S. Picoli, Jr. et al., Europhys. Lett. 75, 673 (2006).

[7] J. E. Iglesias and C. Pecharromán, Scientometrics 73, 303 (2007).

[8] F. Radicchi et al., Proc. Natl. Acad. Sci. U.S.A. 105, 17268 (2008).

[9] H. M. Gupta et al., Braz. J. Phys. 35, 981 (2005).

[10] M. J. Stringer, M. Sales-Pardo, and L. A. N. Amaral, PLoS ONE 3, e1683 (2008).

[11] B. M. Althouse et al., J. Am. Soc. Inf. Sci. Technol. 60, 27 (2009).

[12] J. Laherrère and D. Sornette, Eur. Phys. J. B 2, 525 (1998).

[13] J. E. Hirsch, Proc. Natl. Acad. Sci. U.S.A. 102, 16569 (2005).

[14] P. D. Batista et al., Scientometrics 68, 179 (2006).

[15] A. M. Petersen et al., Europhys. Lett. 83, 50010 (2008).

[16] A. M. Petersen, W.-S. Jung, J.-S. Yang, and H. E. Stanley e-print arXiv:0806.1224.

[17] R. K. Merton, Science 159, 56 (1968).

[18] D. De Solla Price, J. Am. Soc. Inf. Sci. 27, 292 (1976).

[19] J. C. Huber, J. Am. Soc. Inf. Sci. 49, 471 (1998).

[20] R. Albert and A. L. Barabàsi, Rev. Mod. Phys. 74, 47 (2002).

[21] P. L. Krapivsky and S. Redner, Phys. Rev. E 63, 066123 (2001).
[22] B. Freiesleben de Blasio et al., Proc. Natl. Acad. Sci. U.S.A. 104, 10762 (2007).

[23] W. Shockley, Proc. IRE 45, 279 (1957).

[24] J. C. Huber, Scientometrics 45, 33 (1999).

[25] ISI Web of Knowledge, www.isiknowledge.com/

[26] In the ISI database, the author name John M. Doe would be stored as "Doe, J" or "Doe, JM" depending on the author's designation.

[27] M. E. J. Newman, Contemp. Phys. 46, 323 (2005).

[28] A. Clauset, C. R. Shalizi, and M. E. J. Newman, SIAM Rev. 51, 661 (2009).

[29] S. Redner, Am. J. Phys. 58, 267 (1990).

[30] D. Sornette, Phys. Rev. E 57, 4811 (1998).

[31] W. J. Reed and B. D. Hughes, Phys. Rev. E 66, 067103 (2002).

[32] S. Sinha and S. Raghavendra, Eur. Phys. J. B 42, 293 (2004).

[33] J. A. Davies, Eur. Phys. J. B 27, 445 (2002).

[34] A. M. Petersen, O. Penner, and H. E. Stanley e-print arXiv:1003.0134.

[35] C. Castellano et al., Rev. Mod. Phys. 81, 591 (2009).

[36] D. Lazer et al., Science 323, 721 (2009).

[37] M. C. González et al., Nature (London) 453, 779 (2008).

[38] F. Liljeros et al., Nature (London) 411, 907 (2001).

[39] P. Holme et al., Soc. Networks 26, 155 (2004).

[40] R. N. Mantegna and H. E. Stanley, An Introduction to Econophysics: Correlations and Complexity in Finance (Cambridge University Press, Cambridge, England, 1999). 